\title{
Effect of Early Cumulus Cell Removal on the Fertilization and Clinical Outcome in Human in Vitro Fertilization
}

\author{
Jilong Liu ${ }^{1 *}$, Meiyan Chen ${ }^{2 *}$, Chunlian Lin ${ }^{1}$, Xunjin Weng1, Zhongming Meng1, Weijun Tang1 \\ ${ }^{1}$ Assisted Reproduction Technology Center, Qinzhou Maternal and Child Health Hospital, Qinzhou, China \\ ${ }^{2}$ Department of Information Institute of Science and Technology, Qinzhou Science and Technology Bureau, \\ Qinzhou, China \\ Email: ${ }^{*}$ I.i.u.212@163.com
}

Received 11 June 2015; accepted 4 August 2015; published 7 August 2015

Copyright $\odot 2015$ by authors and Scientific Research Publishing Inc.

This work is licensed under the Creative Commons Attribution International License (CC BY). http://creativecommons.org/licenses/by/4.0/

(c) (i) Open Access

\begin{abstract}
The short-term insemination technique has been widely applied, although it is not clear whether the mechanical desorption of cumulus cells can affect normal fertilization and birth outcomes. Therefore, the present study aimed to evaluate whether early cumulus cell removal could affect in vitro fertilization outcomes. Based on a history of infertility and semen analysis, 61 patients were identified, and the cumulus cells were mechanically removed at either6 $h$ post-insemination (Group A, 387 mature oocytes) or 18 - 20 h post-insemination as traditional in vitro fertilization (Group B, 424 mature oocytes). There were no significant differences in the rates of fertilization, available embryos, high quality embryos, birth, premature birth, pregnancy, or implantation when we compared the two groups. However, Group A exhibited a significantly higher polyspermy rate, and significantly lower rates of blastocyst formation and high-quality blastocyst formation, compared with Group B. Therefore, the results of present study indicate that early cumulus cell removal (at $6 \mathrm{~h}$ post-insemination) may negatively affect the rates of polyspermy, blastocyst formation, and high-quality blastocyst formation.
\end{abstract}

\section{Keywords}

Short-Term Insemination, Cumulus Cells, Embryo Quality, Clinical Outcomes

\section{Introduction}

The short-term insemination technique has been widely and successfully applied, and it is gradually replacing

${ }^{*}$ Corresponding author.

How to cite this paper: Liu, J.L., Chen, M.Y., Lin, C.L., Weng, X.J., Meng, Z.M. and Tang, W.J. (2015) Effect of Early Cumulus Cell Removal on the Fertilization and Clinical Outcome in Human in Vitro Fertilization. Advances in Reproductive Sciences, 3, 50-56. http://dx.doi.org/10.4236/arsci.2015.33006 
the conventional human in vitro fertilization (IVF) technique [1]. Demonstrated the time of oocyte exposure to sperm can elevate embryo viability and pregnancy rate by Dirnfeld et al. [1]. Short-term insemination does not affect the fertilization rate and embryo development [2]. In addition, the short co-incubation allows embryologists to observe whether oocyte activation has occurred, and to immediately remedy failed activation. Nevertheless, it is unclear whether the mechanical desorption of cumulus cells can affect normal fertilization or embryonic development potential. For example, various studies have demonstrated that early cumulus cells removal could affect fertilization and embryonic development potential, although contradictory findings are reported [2]-[5]. Moreover, their outcome analysis only examined fertilization outcomes, and did not provide data regarding birth outcomes. Therefore, the effects of early cumulus cell removal remain controversial.

The aim of this study was to investigate the effects of early cumulus cells removal on fertilization, polyspermy, embryonic development potential, blastocyst development, and clinical outcomes.

\section{Materials and Methods}

\subsection{Patients}

A total of 61 patients who underwent short-term insemination were includede in this study. The inclusion criteria were women who were 23 - 38 year old, had failed to achieve pregnancy during at least 5 years of normal sexual activity without contraception, were undergoing their first IVF treatment, and had at least 10 retrieved oocytes. The etiologies for subfertility included tubal disease, endometriosis, ovulatory dysfunction, unexplained, or mixed factors; none of the cases involved exclusively male factors. Among the 61 patients, 5 patients were excluded after receiving early rescue intra-cytoplasmic sperm injection (ICSI) after $9 \mathrm{~h}$ of co-incubation, due to a low fertilization rate or total fertilization failure. Thus, 811 mature oocytes were collected from remaining the patients, and the oocytes were randomly divided into two groups: Group A (387 mature oocytes) underwent early cumulus cell removal at 6 h post-insemination, and the Group B (424 mature oocytes) oocytes were transferred to new sperm-free insemination medium after $6 \mathrm{~h}$ of co-incubation, and the cumulus cellswere removed at 18 - 20 h post-insemination.

\subsection{Controlled Ovarian Hyperstimulation and Oocyte Retrieval}

All patients were treated via routine controlled ovarian hyperstimulation $(\mathrm{COH})$, and gonadotropin releasing hormone agonist was administered for down-regulation in the mid-luteal phase of the menstrual cycle. The patients then received 5000 - 10,000 IU of human chorionic gonadotropin (hCG) when at least three follicles were found to be $\geq 18 \mathrm{~mm}$. All oocyte retrievals were performed via transvaginal aspiration under ultrasound guidance at 36 - $38 \mathrm{~h}$ after the hCG administration.

\subsection{Sperm Preparation and Insemination}

Semen samples were collected via masturbation during the morning of the oocyte retrieval day. Sperm collection was performed using gradient centrifugation. The sperm pellet was then placed at the bottom of fresh insemination medium and incubated at $37^{\circ} \mathrm{C}$ in $6 \% \mathrm{CO}_{2}$ incubators to facilitate the swim-up technique. Next, the supernatant, which contained sperm with strong motility, was collected for insemination. The insemination of the cumulus-oocyte complex was performed via IVF microdroplets, which contained approximately 15,000 50,000 motile sperm in $50 \mu \mathrm{L}$ of insemination medium.

\subsection{Cumulus Cells Removal}

Cumulus cell removal was performed using Pasteur pipettes with an inner diameter of $150 \mu \mathrm{m}$, which is slightly larger than the oocyte. Using this technique, the oocytes were repeatedly aspirated and ejected until the cumulus cells were removed. This technique was performed after $6 \mathrm{~h}$ of co-incubation in Group A, and the oocytes were examined under an inverted microscope at high magnification. Fertilization was defined as the observation of a second polar body, and total fertilization failure was defined as the absence of a second polar body in any of the mature oocytes. Rescue ICSI was performed for fertilization of oocytes that did not exhibit a second polar body after $9 \mathrm{~h}$ of insemination. Patients with a low fertilization rate $(<30 \%)$ or total fertilization failure were excluded from this study. In Group B, the cumulus cells were subsequently removed at 18 - $20 \mathrm{~h}$ after insemination. 


\subsection{Fertilization Assess and Embryo Culture}

Normal fertilization was defined as the presence of two pronuclei, polyspermy was defined as the presence of $\geq 3$ pronuclei, and 1PN oocytes were defined as the presence of one pronucleus. The developmental competence of zygotes with two pronuclei was evaluated after 96 - $120 \mathrm{~h}$ of in vitro culture. During days 1 - 3 post-fertilization, the embryos were placed in G1TM media with 5\% human serum albumin, which was followed by G2TM with $10 \%$ human serum albumin during days 3 - 6 .

Embryo morphology was classified on day 3. Grade I (GI) embryos had even, regular, spherical blastomeres with $<10 \%$ fragmentation. Grade II (GII) embryos had 10\% - 20\% fragmentation and/or unevenly shaped blastomeres. Grade III (GIII) embryos had unevenly shaped blastomeres with $20 \%$ fragmentation. Grade IV (GIV) embryos had unequal, dark blastomeres, with $>20 \%$ fragmentation. Only high-quality embryos (GI-GIII) were used in this study, and two of the high-quality embryos were selected for transfer on day 3 under ultrasound guidance. The blastocysts were assigned a score based on Gardner et al.'s system [6], with the high-quality blastocysts having scores of $\geq 4 \mathrm{BB}$. The surplus high-quality embryos and top-scoring blastocysts were cryopreserved for future transfers.

\subsection{Clinical and Birth Outcomes}

Embryo transfer took place during 3 day after oocytes retrieval, under ultrasound guidance. To reduce the risk of high-rankmultiple pregnancies, the number of replaced embryos was generally limited to two. Luteal support (a combination of estrogen and progesterone) was initiated on day 1 after oocyte retrieval.

The implantation rate was defined as the number of gestational sacs divided by the number of embryos that were transferred. At 35 days post-transfer, the fetal heartbeat was monitored via ultrasonography to confirm the establishment of a clinical pregnancy. At week 20, the details regarding the patient's ongoing pregnancy were recorded via a follow-up phonecall. Delivery before week 37 was defined as a prematurebirth, and a birth weight of $<2500 \mathrm{~g}$ was defined as a low birth weight.

\subsection{Statistical Analyses}

All analyses were performed using SPSS software (version 13.0, SPSS Inc., Chicago, IL). Continuous data were compared using Student's $t$ test, and proportional data were compared using the $\chi^{2}$ test. A P-value of $<0.05$ was defined as statistically significant.

\section{Results}

In Groups A and B, 387 and 424 mature oocytes were collected, respectively (rescue ICSI cycles were excluded in both groups). The patients' demographic data are listed in Table 1, which includes the women's age, duration of infertility, infertility diagnosis, and number of oocytes that were retrieved. However, there were no significant differences in the two groups' demographic data.

The laboratory data for Groups A and B are listed in Table 2. When we compared the two groups, there were no significant differences in the rates of normal fertilization, oocytes with one pronucleus, high-quality embryos, or available embryos. However, a significant difference was observed in the two group's polyspermy rates $(\mathrm{P}<$ 0.01). After transplantation and vitrification on day 3, 151 embryos in Group A were considered low-quality, compared to 147 embryos in Group B; these embryos were transferred to blastocyst medium to continue their culture. These surplus embryos' blastocyst formation and high-quality blastocyst formation rates were significantly lower in Group A, compared to Group B (P < 0.05), after extended culture for 5 or 6 days.

A total of 109 embryo transfers were performed, including 49 embryos in Group A and 60 embryos in Group B (Table 3). A total of 35 patients achieved pregnancy, including 16 patients in Group A and 19 patients in Group B. No significant differences were observed in the rates of clinical pregnancy or birth. The rates of twin/singleton pregnancies and implantation were slightly higher in Group B, compared to Group A, although these differences were not statistically significant.

There were no differences in the sex, twin/singleton births, premature births, and low birth weight status when we compared the two groups (Table 4). However, compared to Group A, Group B had a longer gestational duration $(\mathrm{P}<0.034)$ and a slightly (although not significantly) higher average birth weight. 
Table 1. Demographic data.

\begin{tabular}{cccc}
\hline & Group A & Group B & 31 \\
Patients(n) & 25 & $31.9 \pm 6.2$ & - \\
Women's age (year) & $32.8 \pm 5.6$ & $6.1 \pm 1.8$ & 424 \\
Duration of infertility (years) & $6.2 \pm 2.6$ & 387 & 0.853 \\
No. of mature oocytes & $13.7 \pm 3.6$ & 0.052 \\
\hline
\end{tabular}

Values are presented as $n$ or mean \pm standard deviation. NS, not statistically significant.

Table 2. Laboratory parameters.

\begin{tabular}{cccc}
\hline & Group A & Group B & P-value \\
\hline No. of oocytes & 387 & 424 & - \\
Normal fertilization (\%) & $67.2(260 / 387)$ & $69.1(293 / 424)$ & 0.558 \\
Polyspermy (\%) & $10.9(42 / 387)$ & $5.7(24 / 424)$ & 0.007 \\
1PN (\%) & $1.3(5 / 387)$ & $2.4(10 / 424)$ & 0.260 \\
High-quality embryo (\%) & $42.6(112 / 263)$ & $50.8(152 / 299)$ & 0.051 \\
Available embryo (\%) & $66.2(174 / 263)$ & $71.6(214 / 299)$ & - \\
No. of surplus embryos & 151 & 147 & 0.049 \\
Blastocyst formation (\%) & $16.6(25 / 151)$ & $25.9(38 / 147)$ & 0.028 \\
\hline
\end{tabular}

Values are presented as $\mathrm{n}$ or \%; values were compared using the chi-square test. 1PN, the fertilized ovum has only one pronucleus; surplus embryos, embryos that remained after transplantation and vitrification on day 3; NS, not significant.

Table 3. Clinical pregnancy outcomesafter embryo transfer.

\begin{tabular}{|c|c|c|c|}
\hline & Group A & Group B & P-value \\
\hline Mean embryos per transfer & $1.96(49 / 25)$ & $1.94(60 / 31)$ & 0.946 \\
\hline Clinical pregnancies (\%) & $64(16 / 25)$ & $61.3(19 / 31)$ & 0.835 \\
\hline Implantation (\%) & $40.8(20 / 49)$ & $45.0(27 / 60)$ & 0.661 \\
\hline Twin/singleton pregnancies & $4 / 12$ & $8 / 10$ & 0.236 \\
\hline Ectopic pregnancies & 0 & 1 & - \\
\hline Births (\%) & $48(12 / 25)$ & $48.4(15 / 31)$ & 0.977 \\
\hline
\end{tabular}

Values are presented as n or \%; values were compared using the chi-square test. NS, not significant.

Table 4. Pregnancy outcomesafter embryo transfer.

\begin{tabular}{cccc}
\hline & Group A & Group B & $38.33 \pm 1.35$ \\
\hline Gestational duration (weeks) & $37.17 \pm 1.40$ & $11 / 9$ & 0.034 \\
Male/female & $9 / 6$ & $5 / 10$ & - \\
Twin/singleton & $3 / 9$ & $3308.85 \pm 436.27$ & 3.123 \\
Average birth weight & $3085.33 \pm 387.05$ & 0.656 & $10(2 / 20)$
\end{tabular}

Values are presented as $\mathrm{n}$ or \%; values were compared using the chi-square test. Premature births, delivery before week 37 ; low birth weight, a birth weight of <2500 g; NS, not significant. 


\section{Discussion}

Fertilization is a delicately programmed process that is achieved via numerous cellular and molecular events, which begin at sperm capacitation. After intercourse, the sperm must travel through the cervix to achieve fertilization, although this process can also be facilitated in vitro using culture medium. In conventional IVF, the assessment of fertilization is typically performed at 16 - $20 \mathrm{~h}$ after insemination, although this technique generally achieves low fertilization rates or total fertilization failure inclinical practice. Therefore, after 16 - $20 \mathrm{~h}$ of co-incubation, rescue ICSI is typically performed for unfertilized oocytes, although this technique also typically achieves a poor pregnancy rate [7]. Furthermore, embryos with failed cleavage typically achieve low fertilization rates or total fertilization failure, which can result in cycle cancellation and wasted oocytes, and ultimately reduce the patient's likelihood of achieving pregnancy.

It has been reported that short-term insemination does not affect the fertilization rate, embryo development, and clinical pregnancy rate, although this technique does improve the number and quality of the available embryos [4] [8]. During short-term insemination, the oocyte and sperm are only co-incubated for 1 - 6 h, which allows for the earlier use of rescue ICSI. In contrast, late rescue ICSI (after 16 - 20 h of co-incubation) occurs approximately $24 \mathrm{~h}$ after normal fertilization, and misses the optimal point for fertilization. In addition, prolonged in vitro culture increases the probability of accumulating abnormal genetic material. Furthermore, as the development of the embryo and endometrium is not synchronized, late rescue ICSI may miss the window for optimal endometrial implantation. Thus, early rescue ICSI provides higher rates of fertilization, pregnancy, and birth, compared to late rescue ICSI.

The previously reported rates of polyspermy for conventional IVF and short-term co-incubation are similar [9] [10], although it is not clear whether early removal of the cumulus cells affects polyspermic fertilization. For example, several reports have suggested that excessive short-term insemination does not affect the polyspermic fertilization rate [2] [4] [5]. In contrast, the results of this study showed that short-term co-incubation with early cumulus cell removal significantly increased the rate of polyspermy, although it did not affect the rates of fertilization, usable embryos, or high-quality embryos. Thus, the removal of cumulus cells after $6 \mathrm{~h}$ of co-incubation may have caused abnormal fertilization. In the present study, the rate of polyspermic fertilization was $5.7 \%$ for late cumulus removal, and this result was similar to the findings of Xiong et al. [2]. However, this result conflicts with the findings of Wei et al. [4], who reported no differences in polyspermy when they compared cumulus cell removal after $4 \mathrm{~h}$ (short co-incubation) and 17 - $18 \mathrm{~h}$ (after insemination). Nevertheless, the different study designs may partially explain these conflicting results, as Wei et al. divided their patients into a group with a low risk of fertilization failure (the control group) and a group with a high risk of fertilization failure (the experimental group). In addition, we speculate that the oocyte immaturity may also partially explain the abnormal fertilization rates. Furthermore, the high polyspermic pronuclei rate may be related to the timing of the cumulus cells removal, as it is more difficult to remove cumulus cells before $6 \mathrm{~h}$ post-insemination and the repeated aspiration may also have affected the subsequent fertilization process. As we know, it was harder to remove cumulus cells $4 \mathrm{~h}$ postinsemination compared to cumulus cells removal at $6 \mathrm{~h}$ of insemination. The repeated aspiration may cause damage to cytoplasmic structures and subsequent fertilization process, leading to multiple sperm penetrations, or inhibition of the second polar body extrusion. Moreover, the early observation under microscopes would result in possible temperature and $\mathrm{pH}$ fluctuations during fertilization. Therefore, all of the processes that were described above may have affected the normal fertilization process. Interestingly, specific components of the cumulus cells (e.g., hyaluronic acid) may facilitate sperm capacitation and the acrosome reaction during fertilization [11]. In addition, Suzuki et al. have reported that adding hyaluronic acid to bovine fertilization medium decreased the polyspermic fertilization rate for IVF [12].

The most common method for evaluating embryos is currently morphology scoring. However, the value of embryo morphology on day 3 for predicting subsequent blastocyst formation is limited and inadequate. Therefore, extended human embryo culture may increase the pregnancy and implantation rates, by providing greater discrimination regarding potential embryonic viability. Nevertheless, not all embryos achieve the blastocyst stage, and the cleavage of low-quality embryos during IVF/ICSI-ET may allow for the development of blastocysts; some of these blastocysts have the ability to implant and achieve clinical pregnancy [13]. The factors that can affect blastocyst formation and quality are culture conditions, number of oocytes, maternal age, and male factor infertility. However, little attention has been paid to the effects of early cumulus cells removal on the blastocyst formation. 
Our findings clearly demonstrate that the cleavage of low-quality embryos may indicate future development into blastocysts, as Group B exhibited higher rates of blastocyst formation and high-quality blastocyst formation. This finding likely indicates that the cumulus cells play an important role in modulating human embryo viability. Interestingly, the early stages of embryo development are regulated by the maternal genes [14]. Therefore, before the 8-cell stage, the physiological characteristics are drive by the oocyte, which may be able to repair itself if development is disrupted. Thus, the earliest stage of retarded embryo development may still retain the potential to achieve the blastocyst stage.

In this study, we evaluated the effects of cumulus cells removal at $6 \mathrm{~h}$ and 18 - $20 \mathrm{~h}$ (after short-term insemination), in order to determine the most effective method for improving clinical outcomes. However, only the best quality embryos were transferred (regardless of whether they were derived from the $6 \mathrm{~h}$ group or the 18 - 20 $\mathrm{h}$ group), in order to provide the patient with the greatest chance of pregnancy. Similar to two previous studies, we also confirmed that the two methods provided similar rates of clinical pregnancy and implantation. We also performed further analyses to more clearly distinguish these two methods. Using these two methods, Groups A and B achieved 12 and 15 live births, respectively, although the gestational duration in Group A was lower than that in Group B. One possible explanation for this discrepancy is the very small number of cases. However, the two groups exhibited no significant differences in the other birth outcome parameters, such as birth rate, average birth weight, low birth weight, twin/singleton pregnancies, or sex ratio.

\section{Conclusion}

In conclusion, the results of present study suggest that early cumulus cells removal at $6 \mathrm{~h}$ post-insemination may increase the polyspermy rate and decrease the blastocyst quality and viability of low-quality embryos. However, the optimal conditions for in vitro embryo development mimic spontaneous fertilization, and avoid manual interventions if possible. Therefore, further prospective randomized trials are needed to compare the effectiveness of different fertilization methods.

\section{References}

[1] Dirnfeld, M., Bider, D., Koifman, M., Calderon, I. and Abramovici, H. (1999) Shortened Exposure of Oocytes to Spermatozoa Improves In-Vitro Fertilization Outcome: A Prospective, Randomized, Controlled Study. Human Reproduction, 14, 2562-2564. http://dx.doi.org/10.1093/humrep/14.10.2562

[2] Xiong, S., Han, W., Liu J.X., Zhang, X.D., Liu, W.W., Liu, H. and Huang, G.N. (2011) Effects of Cumulus Cells Removal after 6 h Co-Incubation of Gametes on the Outcomes of Human IVF. Journal of Assisted Reproduction and Genetics, 28, 1205-1211. http://dx.doi.org/10.1007/s10815-011-9630-9

[3] Chen, C. and Kattera, S. (2003) Rescue ICSI of Oocytes That Failed to Extrude the Second Polar Body 6 h Post-Insemination in Conventional IVF. Human Reproduction, 18, 2118-2121. http://dx.doi.org/10.1093/humrep/deg3253

[4] Wei, D., Zhang, C.L., Yin, B.L., Wang, P., Xie, J.K., Song, X.B., Liu, Q., Hu, L., Zhang, Y.X. and Hao, H.Y. (2011) Early Cumulus Cell Removal Could Reduce the Available Embryo Rate in Human IVF. Journal of Assisted Reproduction and Genetics, 28, 1213-1216. http://dx.doi.org/10.1007/s10815-011-9650-5

[5] Xue, Y.M., Tong, X.M., Jiang, L.Y., Zhu, H.Y., Yang, L.Y. and Zhang, S.Y. (2013) Effect of Cumulus Cell Removal 4 h Post-Insemination on Fertilization and Embryo Quality: A Prospective Randomized Sibling-Oocyte Study. Journal of Assisted Reproduction and Genetics, 30, 1049-1053. http://dx.doi.org/10.1007/s10815-013-0049-3

[6] Gardner, D.K., Lane, M., Stevens, J., Schlenker, T. and Schoolcraft, W.B. (2000) Blastocyst Score Affects Implantation and Pregnancy Outcome: Toward a Single Blastocyst Transfer. Fertility and Sterility, 3, 1155-1158. http://dx.doi.org/10.1016/S0015-0282(00)00518-5

[7] Morton, P.C., Yoder, C.S., Tucker, M.J., Wright, G., Brockman, W.D. and Kort, H.I. (1997) Reinsemination by Intracytoplasmic Sperm Injection of 1-Day-Old Oocytes after Complete Conventional Fertilization Failure. Fertility and Sterility, 68, 488-491. http://dx.doi.org/10.1016/S0015-0282(97)00223-9

[8] Kattera, S. and Chen, C. (2003) Short Coincubation of Gametes in in Vitro Fertilization Improves Implantation and Pregnancy Rates: A Prospective, Randomized, Controlled Study. Fertility and Sterility, 80, 1017-1021. http://dx.doi.org/10.1016/S0015-0282(03)01154-3

[9] Pieters, M.H., Dumoulin, J.C., Ignoul-Vanvuchelen, R.C., Bras, M., Evers, J.L., Geraedts, J.P. (1992) Triploidy after in Vitro Fertilization: Cytogenetic Analysis of Human Zygotes and Embryos. Journal of Assisted Reproduction and Genetics, 9, 68-76. http://dx.doi.org/10.1007/BF01204118 
[10] Gianaroli, L., Cristing Magli, M., Ferraretti, A.P., Fiorentino, A., Tosti, E., Panzella, S. and Dale, B. (1996) Reducing the Time of Sperm-Oocyte Interaction in Human in-Vitro Fertilization Improves the Implantation Rate. Human Reproduction, 11, 166-171. http://humrep.oxfordjournals.org/content/11/1/166.long

[11] Fatechi, A.N., Zeinstra, E.C., Kooij, R.V., Colenbrander, B. and Bevers, M.M. (2002) Effect of Cumulus Cell Removal of in Vitro Matured Bovine Oocytes prior to in Vitro Fertilization on Subsequent Cleavage Rate. Theriogenology, 57, 1347-1355. http://dx.doi.org/10.1016/S0093-691X(01)00717-8

[12] Suzuki, K., Eriksson, B., Shimizu, H., Nagai, T. and Rodriguez-Martinez, H. (2000) Effect of Hyaluronan on Monospermic Penetration of Porcine Oocytes Fertilized in Vitro. International Journal of Andrology, 23, 13-21. http://dx.doi.org/10.1046/j.1365-2605.2000.t01-1-00198.x

[13] Balaban, B., Urman, B., Alatas, C., Mercan, R., Aksoy, S. and Isiklar, A. (2001) Blastocyst-Stage Transfer of PoorQuality Cleavage-Stage Embryos Results in Higher Implantation Rates. Fertility and Sterility, 75, 514-518. http://dx.doi.org/10.1016/S0015-0282(00)01756-8

[14] Braude, P., Bolton, V. and Moore, S. (1988) Human Gene Expression First Occurs between the Four- and Eight-Cell Stages of Preimplantation Development. Nature, 332, 459-461. http://dx.doi.org/10.1038/332459a0 